\title{
Abdominal Mass Caused Failure to Thrive in a Young Boy: Mixed-Type Localized Retroperitoneal Castleman Disease
}

\author{
Bahar Ashjaei $^{a} \quad$ Afshar Ghamari Khameneh $^{\mathrm{b}} \quad$ Gisoo Darban Hosseini Amirkhiz ${ }^{\mathrm{c}}$ \\ aDepartment of Pediatric Surgery, Pediatric Center of Excellence, Tehran University of Medical \\ Sciences, Tehran, Iran; ${ }^{b}$ Department of Radiology, Faculty of Medicine, Mashhad University of \\ Medical Sciences, Mashhad, Iran; 'Research Center for Prevention of Cardiovascular Disease, \\ Institute of Endocrinology and Metabolism, Iran University of Medical Sciences (IUMS), Tehran, \\ Iran
}

Keywords

Castleman disease $\cdot$ Retroperitoneum $\cdot$ Pediatric $\cdot$ Failure to thrive $\cdot$ Anemia

\begin{abstract}
Castleman disease is a rare cause of retroperitoneal mass in children. Clinical presentation and laboratory findings are usually nonspecific. Imaging shows features of a hypervascular or soft tissue mass. We present a 12-year-old boy who complained of frequent colds, fatigue, and failure to gain weight for the past 4 years who was referred to our hospital. Anemia and hypergammaglobulinemia were revealed in laboratory tests. Imaging showed a well-delineated retroperitoneal soft tissue mass with intense homogenous enhancement at the midline below the aortic bifurcation. The histopathological features were consistent with mixed type unicentric Castleman disease. Surgical removal was curative. Our patient's hematological abnormalities resolved, and he gained $10 \mathrm{~kg}$ in the next 4 weeks after the operation and reached the 25-50th percentile for his age.

\section{Introduction}

Castleman disease (CD) is a rare benign cause of nonneoplastic lymphadenopathy. The etiology is poorly understood. Clinically, it is classified into unicentric (localized) and multicentric (disseminated) [1]. Unicentric CD may occur at any age. It is rarely found in the pediatric population, though. The mediastinal lymph nodes (70\%) are most commonly affected. The retroperitoneum is an infrequent site of localized disease [2,3]. CD patients are usually asymptomatic or have nonspecific abdominal or back pain. Laboratory abnormality or constitutional symptoms may be found in a small portion [4]. 


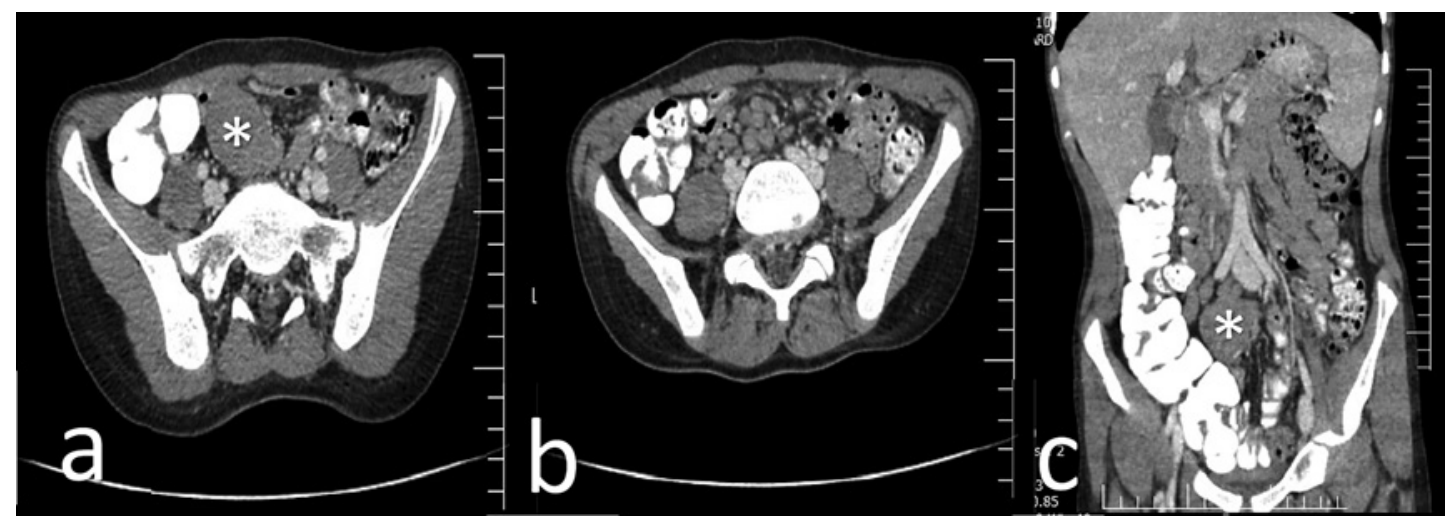

Fig. 1. a Axial CT scan, there is a retroperitoneal homogeneously enhancing mass $\left({ }^{*}\right)$ with well-defined borders. b Multiple satellite small lymph nodes are noted. c A sagittal reformatted CT scan confirmed the position of the mass $\left(^{*}\right)$ below the aortic bifurcation.
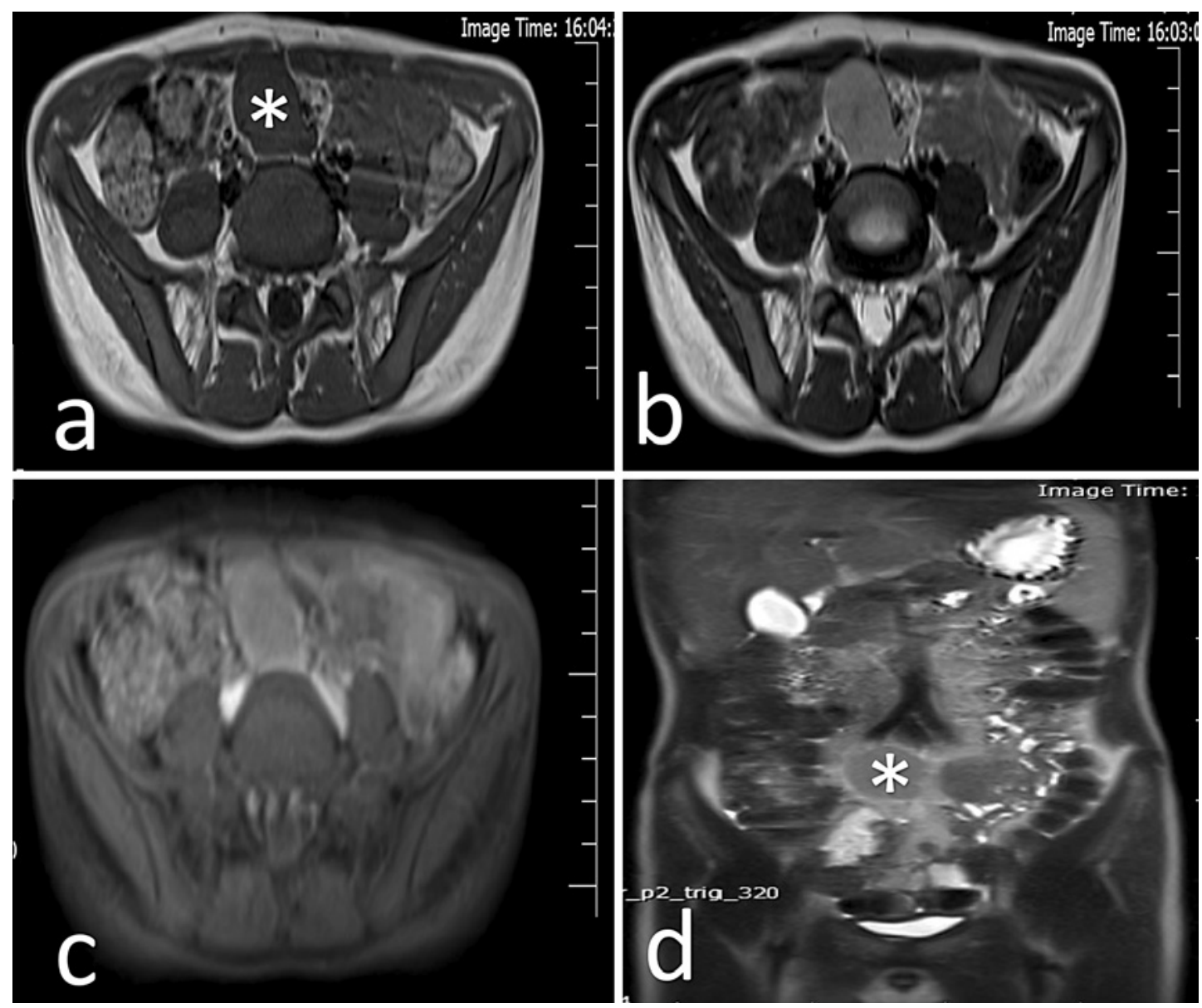

Fig. 2. An abdominal MRI demonstrated a well-circumscribed homogenous retroperitoneal mass $(*)$, isointense on T1-weighted image (a) and hyperintense on T2-weighted image (b) with enhancement $(\mathbf{c}, \mathbf{d})$. 
Ashjaei et al.: Abdominal Mass Caused Failure to Thrive in a Young Boy

\section{Case Report}

A 12-year-old boy who complained of frequent colds, fatigue, and failure to gain weight for the past 4 years was referred to our hospital. He did not state decreased appetite. On physical examination, he had a pale appearance. His height and weight were between the 10 and 25 th and $<5$ th percentiles for his age, respectively. No palpable abdominal mass was detected.

Laboratory evaluations presented anemia of chronic disease for the last 4 years associated with an elevated erythrocyte sedimentation rate, C-reactive protein, and gamma globulins (IgG: 2,083 [normal range: 462-1,682], IgE: 425 [normal range: 38-251]). The laboratory findings were otherwise unremarkable.

On abdominal ultrasound, a hypoechoic solid mass was depicted in the para-aortic region with multiple surrounding hypoechoic small lymph nodes. For better evaluation, an abdominal CT scan with contrast media was performed. There was a well-delineated $38 \times 27 \times 30 \mathrm{~mm}$ retroperitoneal soft tissue mass with intense homogenous enhancement at the midline below the aortic bifurcation. Some small mesenteric and para-aortic lymph nodes around the soft tissue mass were detected (Fig. 1). MRI was also done (Fig. 2). Conglomerated lymph nodes and lymphoma were considered in the differential diagnosis of the main lesion.

No other abnormalities were found in other body regions including the thoracic and axillary regions. Excisional biopsy through laparotomy was performed.

The histopathological evaluation showed an enlarged lymph node with marked lymphoid hyperplasia. Sheets of plasma cells around enlarged lymphoid follicles and the presence of hyalinized material in some areas were also noted. These features were consistent with mixed-type unicentric CD.

The patient recovered well after surgical removal of the mass and was discharged. Mesenteric and para-aortic lymph nodes no longer existed on a follow-up CT scan. The hematological abnormalities resolved, and he gained $10 \mathrm{~kg}$ in the next 4 weeks after the operation and reached the 25-50th percentile for his age.

\section{Discussion}

Localized retroperitoneal CD is a rare differential diagnosis of an abdominal mass, especially in children. CD normally has an indolent clinical course with benign biological behavior. In previously reported cases, the patients' age ranged from very young to elderly individuals. Generally, patients are asymptomatic or clinical presentations are nonspecific. Slowly progressing enlargement of the affected lymph node may be revealed [3].

Diagnosis based on imaging findings is challenging. Affected lymph nodes are sharing features with a hypervascular mass lesion. They are typically homogenous with well-defined borders in different modalities. On CT scans, they show soft tissue attenuation with enhancement following contrast administration. If the measured mass is $>5 \mathrm{~cm}$, heterogenicity may be found. They are iso- to slightly hypointense on T1-weighted images and hyperintense on T2-weighted images. Small satellite mesenteric lymph nodes and mass effect on surrounding structures may be detected $[4,5]$.

There is no specific clinical presentation or imaging feature. Thus, mesenteric lymph node resection followed by histopathological examination is inevitable. Histologically, the disease is subdivided into 3 subtypes in order of decreasing frequency, including hyaline vascular, plasma cell, and mixed [1, 2]. Anemia, hypergammaglobulinemia, hypoalbuminemia, and constitutional symptoms including weight loss, failure to thrive, fever, and weakness are mostly found in plasma cell or mixed subtypes, and in patients with clustered lymph nodes [4].

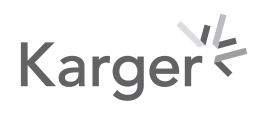


Complete surgical removal is curative in localized CD. After the surgery, constitutional symptoms, hematological abnormalities, and regional satellite lymph nodes will be eliminated. Long-term outcome is excellent but there is evidence of an association with lymphoma, so close follow-up is necessary in all CD patients $[6,7]$.

\section{Conclusion}

Localized retroperitoneal $\mathrm{CD}$ is an infrequent cause of an abdominal mass in children, but associated satellite lymph nodes, constitutional symptoms, laboratory findings, and a hypervascular mass on imaging should raise suspicion in clinicians.

\section{Statement of Ethics}

Formal written consent was obtained from the patient's legal guardian (his father in this case as the patient was under 18 years). The patient's father has agreed to the publication of this case report and accompanying images. The patient's father has given oral and written consent for the use of radiological and pathological images, and he is aware that the report is published in a medical journal which is distributed worldwide for educational purposes.

\section{Conflict of Interest Statement}

The authors have no conflicts of interest relevant to this article to disclose.

\section{Funding}

The authors did not receive any funding support.

\section{Author Contributions}

All authors have been involved in the paper's conception, data acquisition, and manuscript drafting.

\section{References}

1 Cronin DM, Warnke RA. Castleman disease: an update on classification and the spectrum of associated lesions. Adv Anat Pathol. 2009 Jul;16(4):236-46.

2 Keller AR, Hochholzer L, Castleman B. Hyaline-vascular and plasma-cell types of giant lymph node hyperplasia of the mediastinum and other locations. Cancer. 1972 Mar;29(3):670-83.

3 Simpson D. Epidemiology of Castleman disease. Hematol Oncol Clin North Am. 2018 Feb;32(1):1-10.

4 Wong RSM. Unicentric Castleman disease. Hematol Oncol Clin North Am. 2018 Feb;32(1):65-73.

5 Kim TJ, Han JK, Kim YH, Kim TK, Choi BI. Castleman disease of the abdomen: imaging spectrum and clinicopathologic correlations. J Comput Assist Tomogr. 2001 Mar-Apr;25(2):207-14.

6 Bucher P, Chassot G, Zufferey G, Ris F, Huber O, Morel P. Surgical management of abdominal and retroperitoneal Castleman's disease. World J Surg Oncol. 2005 Jun 7;3:33.

7 Talat N, Belgaumkar AP, Schulte KM. Surgery in Castleman's disease: a systematic review of 404 published cases. Ann Surg. 2012 Apr;255(4):677-84.

\section{Karger'₹}

